\title{
Gastrostomía percutánea endoscópica en lactantes con daño neurológico severo
}

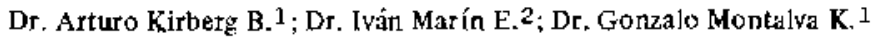 \\ Percataneous endoscopic gastrostomy in infants \\ with severe neurological impairment
}

\begin{abstract}
Percutaneous endoscopic gastrostomy (PEG) is a tichnique to consiruct a feeding gastrostomy without need of general anestlesia nor laparotomy. We have performed two of this procedures in infants, aged 3 and 16 months, with severe neurological inpairment: one of congenital origin and other secondary to perinatal hypoxia. Buth procedures were carricd out at the endoscopy toom and caused minimal disconfort to the patients. There were no complications and only a few minutes ( 7 and 8 respectively) wete reculired. During the follow up of thesc patients ( 7 and 4 months) there was not wound infection around the tube nor meal rellux, nutritional condition inproved and no respiratory troubles occurred. PLG is a safe, simple unexpensive technique wich allows cntcral feeding for long periods in infants with severe neurological impaimont.
\end{abstract}

(Key words: gastrostomy, petcutaneous).

La gastrostomia percutánea endoscópica (GPE) es un procedimiento para instalar una sonda de gastrostomia sin necesidad de laparototnía ni de anestesia general. ${ }^{1}$ Se ha utilizado principalmente en pacientes que no pueden tragar por daño neurológico sevoro o tumores de cabeza y uelio.2 También se la indicado para descompresión gástrica prolongada, como tratamicnto definitivo del vólvulo gástrico y para reintegrar la bilis al tubo digestivo en pacientes portadores de un drenaje biliar externo. ${ }^{3.6} \mathrm{Pre}$ sentamos dos casos de lactantes oon dajo neuro. lógico severo, uno perinlatal y cl otro congénito. en los cuales hemos efectuado este procedimien. to.

\section{CASOS CLINICOS}

1. Lactante de sexo indsculino stegunde lijo de nadse de 20 años y padse de 21 años, vanos. Fmbarazo cursó con allerlia sivera, parto espontánto, peso ajaci-

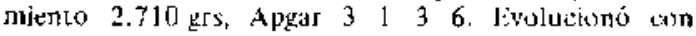

1. Unidad de Gastroenterologia, Hospital Regional de: lquique.

2. Servicio de Pediatria, Hospital Regional de Iquicque. scvesa dificultad Ispiratoria que hieo nectsario apoyo con ventilación mecinica por l semana (IPPB-CPAP). Posterormente tuvo manitestaciones slinicas de encelialupatía hipóxica isquénica. espasticidad acentuada de: extremidades inferiores y ausionsia de reflejo de succión. La ecoencefalografia mostró hidrocefalia leve do veotrículos laterales y tereer ventrículo y el examen oftalmológico atrofia bilateral de papilas. Fue alimentada por sonda nasogístries Jográntose ur ascenso pondera] adecuads. No succionaba dorante los primeros thests y el estado nearolígico se mantuso estacionario por lo

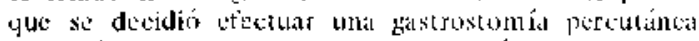
endoscópica, a la edad de tres meses, dindose de alta? sentallas dispuis.

2. Secundal hija de nudre de 23 años y pudre de ?9 años, whos. 1:mbatatzo y pertos normales. Peso al nacier 2.780 g. Apgate 847 . Ictericia nconatal con hilirrabimoma máxima de 13 mojd L. Latancia cxelusiva por

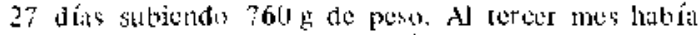
poco progetso dol peso, ingresó a CONIN dond lue

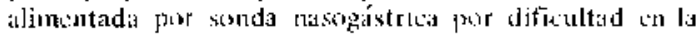

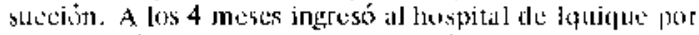
neumluparia secundarial a aspiraciór de leche, veverer cotardo sicomotor y disnutrición gradis 2. Se derivó a Santiago donde los estendios concluyeren tque satria

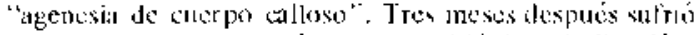

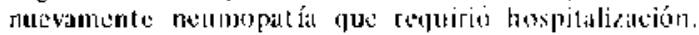
Por la dificultad permancule de succión la comencia a tener stwerom cuadros respiratorios se realizá gastrostar

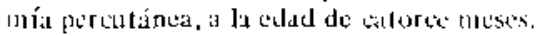

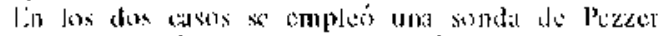
N'I4 enamblándola on una cámula tondovenosia

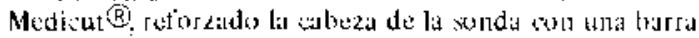

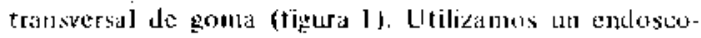




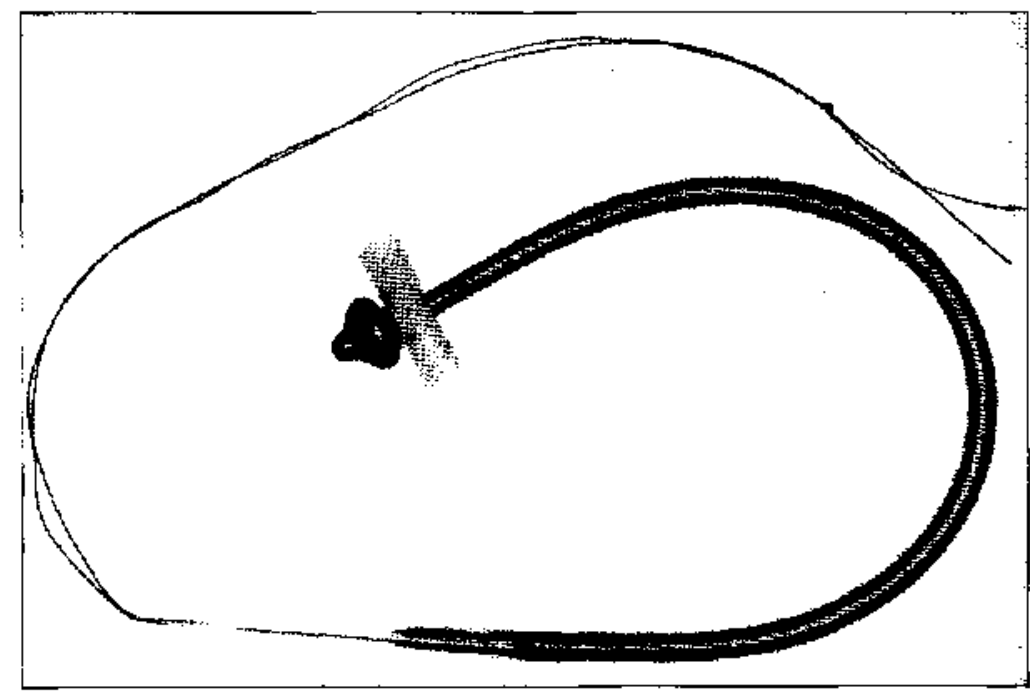

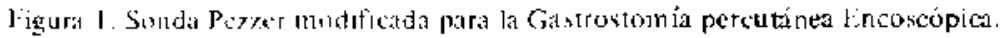

pio Olymusu Grle $Q$ desunfectado en gluturaldehido al 5\% rour pa de hopsia csterilizada en gas.

las paticntes tueron preparados con suspensión de la abmentación poz sonda misogútstica 12 horas antes del procediuniento, aseo proligo de la cavidad orill con povidona yodada y una primesa dosis de exalosporina profilictici.

Los dos procedimjentos se efectuaron th la sala de endoscopía del hospital. No ste emples anestes]a gencral ní premedicación. Se requirió la participación de un endoscopista cun experiencia pediátrica, un cirujano y unj enfermera.

Con el miño bien inmovilizado, ic exumina endosópicamente esófiago, estumitgs y duudeno. Se insutha el estómago para que wotacte con la pared abdominat, desplazando el hígado y al colon tranverso. Se prepara Ia pared abdominal con tintura antiséptica $y$ paijos estériles. Con la luz de la salla apagada se eligo el punto de punción en forma coordinada. il endoscopio ilumina el sitio jdeal sobre el ángulo gústrico y el cirujimo deprime csta zona iluminada con el dedo. Anestesia local e incisión de la piel de aptoximadamente $5 \mathrm{~mm}$. Punción gástrica con cárula endovenos Medicut ${ }^{(B)}$. Se introduce al estónago, a través de la cinula, el extremo de una sedu $\mathrm{N}^{\circ} 2$ de $80 \mathrm{~cm}$ que se coge con la pinza de biopsia y exterioriza por la boca al retirar el endoscopio. Se retira también la cánula quedando el niño "cnhebrado" con un extremo de la seda emorgienulo por la pared abdominal $y$ el otro por la boca. En el extremo bucal se ata fimemente la sonda de gastrostomía ya ensamblada, estérìl y lubricada. Ejerciendo tracción por el cabo abdominal se arrastra la sonda por su extremo proximal por esófago y estómago hasta emerger por la pared abdominal. Uha segunda endoscopía indicará al cirujano cuanto debe traccionar la sonda para lograr una adhe sión adecuada cntre la parcd gástrica y la pared abdominal. Una segunda barra atravesada se destza por la sonda hasta la piel y se fja mediante una gota de cianoacrilato

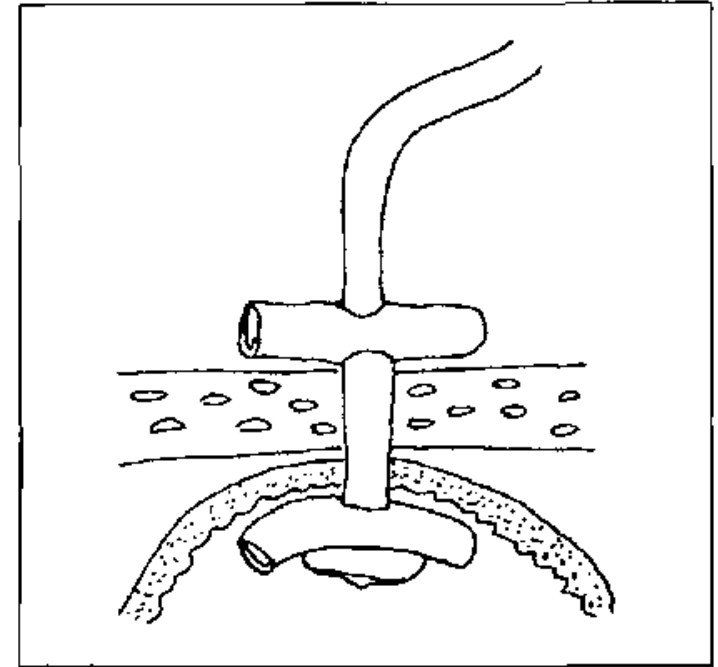

Figura 2. Ėl estómago se mantiene adosado a la paced abdominal entre dos barras transversales. La externa se fija a la sonda con una gota de cianoacrilato para evitar poner puntos.

entre sonda y barra. Fsto impedirá que la sonda avance hacia el píloro levada por el peristaltismo, (f igura 2 y 3). La alimentación se reinicia a las 24 horas y los pacientes son controlados por el mismo equipo hasta su alta.

En los dos casos el procedimiento se realizó sin dificultades, demoró 7 y 8 minutos. Los dos lactantes se realimentaron al día siguiente por esta nueva vía, han completado 7 y 4 meses deste el procedimiento, no han presentado reflujo gastroesofágico de la papilla ni infec- 


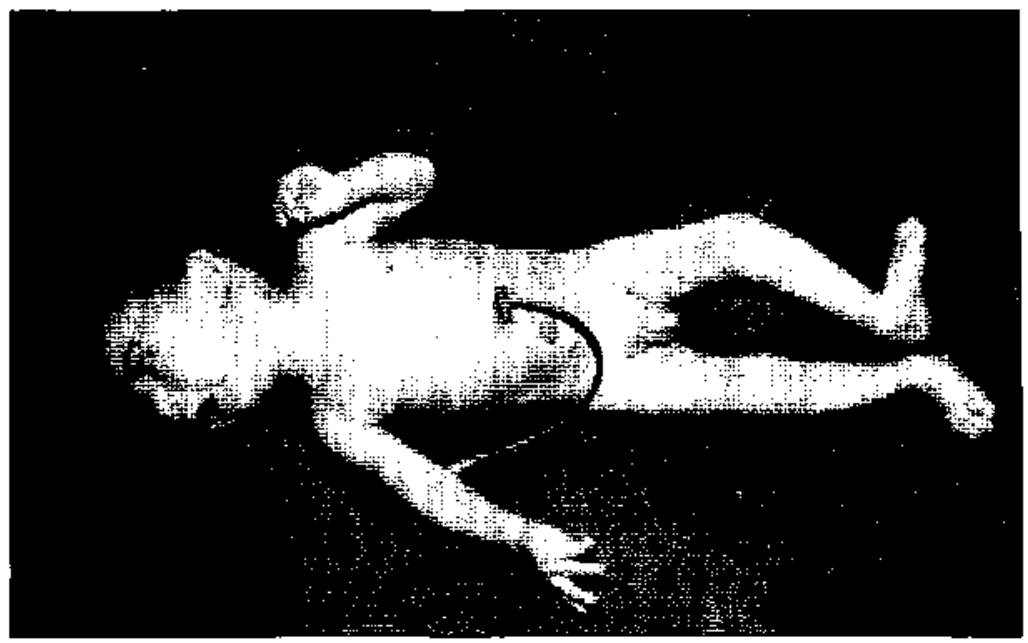

Figura 3. Gastrostomía percutínę endoscópica en lactante de 4 meses con daño neurológico severo perinatal.

ción del sitio de punción. Ambos han dexarsollado tej ido de neolurniación, en torno is la sonda, cuyo estudio histológico reveló que se trataba de granujomas. Estos regresaron luego de tocaciones con nitrato de plata.

\section{COMENTARIOS}

Hemos efectuado gastrostumia percutánca cndoscópica a dos lactantes con daño ncurologico que no les permitía alimentarse por boca. No hemos tenido complicaciones en estos dos casos, que fucron precedidos por 22 procedimicntos en adultos. 6 Las complicaciones que se lan comunicido con esta técrica sun pooco trecuentes, pero es importante consideraras al indica el procedimiento: inficción en torno a la sonda, reflujo gastroesutägico de la pa pilial. neuInoperitonco postoperatorio y salidat accidental de la sonda. También xe ha descrite an easo de fístula gastrocólica, en an niño, la que aparecio? moses después del procediniento ${ }^{3} y$ cedió expont táneamente al retirar la sonda. la compliciciont más severa ocurrió en dos adultos. ennsistio en faceitis necrotizante, y causó la muerte de uno de ellos. ${ }^{7}$ Con el propósito de preven ir las complica. ciones infecciosas hemos adoptado un protocolo especílico en estos dos casos pediátricos, que consiste en realizar la técnica en las mejores condiciones posibles de asepsia, $y$ usar antibiót $i$ cos profilácticos. El neunoperitoneo es habitual y no tiene importancia clínica. ${ }^{9}$ Para prevenir la salida accidental de la sonda debe evitarse excesj. va tensión de ésta al instalarla: si la complicación ocurre culos 15 dias siguientes al procedimiento deberá reinstalarse la sonda, lo más pronto posible, repitiendo la técnica: si ocurre después, cuando ya se ha formado un trayecto fibroso, la reinstalación es simple y no requicre endoscopia. Cuando hay reflujo de la papilla se debe transformar la GPE en yeyunostomia percutánea endoscópica, procedimiento simple, descrito por varios autores. ${ }^{9}, 10$ Está claramente demostrado que la somda uasogástrica tüda por tiempo prolongado provoca serios trastornos respiratorios y daño de la mulcosa esofásica al tavorecer el reflujo gastrocsolagico. La sonda fina de silastic provocaría menos reflujs y menos molestias al paciente, pero liene tendencis a ocluirse, su costo es clevader y duration limitada. La sonda de gastros. comait preparadia como lo hemos descrito, resulta muy connomicia, puede durar más de un año. su recambio es simple y su fumen amplio parmite administrat culalcuier tipo de papilla, comercial o cuscria. Tiene también la ventaja de disimularse bajo la rupa. lo que resulta importante para un nino que mantiene contacto social y para la nitdre que sale con él en brazos. Es dificil en nucstros casos, saber si estos niños aprenderán a alimentarse por boca. Sus madres le ofrecen constantcmente alimentos por esa vía con la esperanza de que algún día lo lograrán. De succder así se mantendrá cerrada la sonda hasta asceurarse de que no es necesaria y luego se retirara cjerciendo una tracción continuada. Esto 
Io hemos experimentado en 5 pacientes adultos que recuperaron lit capacidad de alimentarse entre 3 y 7 meses después de instalada la sonda. Creenos que la GPE es un procedimiento simple. seguro y de gran utilidad para aquellos nifios con daio neurolígion perinatal o wongénito, que requiesen de ana via de alimentación enteral por tiempo prokngado.

\section{RESUMEN}

La sastrostomia pereutanea endoscopica (CPE) es una técnica pera instalat una gastrostomí de alimentación sin necesidald de laparoto. mia ni anestesia general. Hemos efect 1 do dos de estos procedimicutos en lactantes con daino neu. rológico severo, llite congénito y el at ro secundario a hipoxia perinatal. Ambos procedimientos. realizados en la sala de endoscopía, resultaron simples y rápidos ( 7 y 8 minutos) provocando minimas molestias a los pacientes, Los lactantes llevan 7 y 4 meses aliment andose por esta nucva via. No han presentado infección de la herida en torno a fa sonda ni reflujo gastroesufágicir de la papilla. Han mejorado notablemente su estado nutritivo y no lan vuelto a presentar trastornos respiratorios. Creenos que la GPE es una técnica segura, simple y de bajo costo que permite la alimentación enteral por largos períodos en lac. tantes ton daño neusológico seveso.

\section{REFERENCIAS}

1. Cotrdorer W.w.L., Runsky J.L., Izont R.L... Gastrostomy without laparotony: a percutanous endoscopic technique. I Pediat Surg 1980: 15: 872.

2. Rupprnt $H_{\text {, }}$, Lux $G_{n}$ : Percutaneous Findoscopic Gastrostouny in patients with Houd and Neck Cancer. Iinsluscopy. 1986:18:149.

3. Pansky J.t. Ciatuteror M. W.L.. Sfellato T.A.: Percutaneous endrscopic gastrostomy. A review of 150 ases. Arcl Surg 1983:118:913.

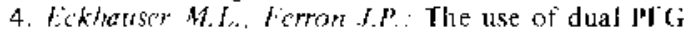
(DPF) in the management of chonic intermitent gastric volvulus. Gastointest fendresc. 1965: 31 : 340.

5. Ponxkl J.L., Aszodi A: Ixternal biliary-gustric fistula: a simple method for recycling bile. Am J (iastrontertil 1982: 77:939.

6. Kibberg A.. Crw S. Gilstrosionía Percutánea Endoscripicat. Rev Méd Chile. 1986;114:832.

?. (ircif J.H., Raglond J.J. Ochsurer M.C. et al: Fatal Ntcrotizing Fasciètis following PI:G. Gastrointest lindose 1986; 32; 253.

8. Fottified E.B. Pfumser A.8., Chir M.R.: Pncumoperitoncum following PEG. Gastrointest Lidosc 1986: 32: 397.

9. Strodel W.E., Eckhouser F.E., Dent T.L. Lemmer I.K. Gastrostomy to yeyunostomy conversion, Gastrointes Endose 1984; 30: 35.

10. Wadieala I.M., Bacon B.R.: A simplified technique for constructing a feeding yeyunostomy from an existing gastrostomy. Gastrointest Endosc 1986; $32: 288$. 